\title{
ANÁLISE DOS NÍVEIS DE IMUNOGLOBULINAS SÉRICAS E MONÓCITOS DE PACIENTES EM TRATAMENTO COM AUTO-HEMOTERAPIA
}

\author{
Adriane Cordeiro Trevisani $1^{1}$ \\ Catchia Hermes-Uliana² \\ Celso Yukimasa Obikawa ${ }^{1}$ \\ Elza Teruko Nishitani ${ }^{1}$ \\ Aparício Luciano Bolonhez ${ }^{3}$ \\ Sandra Mara Alessi Aristides ${ }^{4}$
}

TREVISANIL, A. C.; HERMES-ULIANA, C.; OBIKAWA, C. Y.; NISHITANI, E. T.; BOLONHEZ, A. L.; ARISTIDES, S. M. A. Análise dos níveis de imunoglobulinas séricas e monócitos de pacientes em tratamento com auto-hemoterapia. Arq. Cienc. Saúde UNIPAR, Umuarama, v. 19, n. 2, p, 101-107, maio/ago. 2015.

RESUMO: A auto-hemoterapia (AHT) é uma prática de uso clínico crescente, que vem sendo utilizada como tentativa no tratamento de diversos problemas de saúde, tanto em humanos como em animais. Esse estudo teve como objetivo avaliar o efeito da AHT por meio da avaliação dos níveis de imunoglobulinas e de monócitos de pacientes que realizaram pela primeira vez o tratamento. Foi realizada a coleta de sangue antes e após 24 horas da realização da AHT, e também durante nove dias consecutivos em dez participantes, sendo cinco homens e cinco mulheres. Para realização da AHT foi coletado $10 \mathrm{~mL}$ de sangue venoso e aplicado no quadrante látero-superior do músculo glúteo máximo. Dos dez participantes analisados nove apresentaram aumento do número de monócitos que variou de $17 \%$ a $250 \%$. Observou-se também aumento da Imunoglobulina $\mathrm{M}$ em nove dos indivíduos estudados, que variou de 10 a $28 \%$. O aumento da Imunoglobulina $\mathrm{G}$ foi demonstrado em todos os participantes e variou de 13 a 36\%. Foi observado também aumento de 2,6 a 89\% da Imunoglobulina A em nove pacientes e aumento de 2,7 a $88,2 \%$ da Imunoglobulina $\mathrm{E}$ em oito indivíduos. Conclui-se que a auto-hemoterapia é fator de incremento da imunidade do organismo, pois mostrou aumento do número de monócitos e de imunoglobulinas.

PALAVRAS-CHAVE: Auto-hemoterapia; Imunidade; Sistema imune; Terapias complementares.

\section{ANALYSIS OF SERUM IMMUNOGLOBULIN LEVELS AND MONOCYTES IN PATIENT TREATED WITH AUTOHEMOTHERAPY}

\begin{abstract}
Autohemotherapy (AHT) is a practice that has increasing clinical use, which is being used in an attempt to treat various health problems in both humans and animals. This study aimed to evaluate the effect of AHT by evaluating the levels of immunoglobulins and monocytes of patients who underwent the treatment for the first time. Blood was collected before and 24-hours after the AHT was performed, and also for nine consecutive days, in ten participants, five men and five women. For the AHT, a total of $10 \mathrm{~mL}$ venous blood was collected and applied to the lateral-upper quadrant of the gluteus maximus muscle. From the ten participants analyzed, nine presented an increased number of monocytes, ranging from $17 \%$ to $250 \%$. An increase in immunoglobulin $\mathrm{M}$ was observed in nine of the study subjects, ranging from 10 to $28 \%$. An increased Immunoglobulin $\mathrm{G}$ was shown in all participants, which ranged from 13 to $36 \%$. An increase of 2.6 to $89 \%$ in immunoglobulin A was observed in nine patients, and an increase of 2.7 to $88.2 \%$ in immunoglobulin E in eight individuals. It can be concluded that autohemotherapy is a factor of increased immunity of the body, since it showed an increase in the numbers of monocytes and immunoglobulins.
\end{abstract}

KEYWORDS: Autohemotherapy; Immunity; Immune system; Complementary therapies.

\section{Introdução}

A auto-hemoterapia (AHT) é uma técnica antiga, utilizada em diversas doenças infecciosas, alérgicas e auto-imunes (REIMANN; HAVENS, 1990). Considerada um tratamento controvertido, porém bem sucedido, usado especialmente em países da Europa (GEOVANINI; NORBERTO, 2009). Inicialmente conhecida como auto-hemotransfusão, foi introduzida por Ravaut em 1913, sendo então empregada para vários tipos de doenças sistêmicas de origem desconhecida (RAVAUT, 1913). Foi utilizada em uma variedade de doenças com resultados encorajadores (METTENLEITER, 1936). No Brasil, o difusor da AHT foi o Dr. Luiz Moura, médico e usuário do tratamento, que se baseou nos estudos de Teixeira (1940) e Veronesi (1976), e introduzindo o método em sua prática clínica, relatando inúmeros casos de sucesso obtido com o uso do procedimento em um DVD lançado no ano de 2006, que se difundiu rapidamente por todo o Brasil. $\mathrm{O}$ tratamento visa à auto-estimulação do sistema imunológico pela retirada de determinado volume de sangue venoso e aplicação deste mesmo volume por via intramuscular. É considerada técnica simples que estimula o aumento dos macrófagos (monócitos) pela medula óssea (GEOVANINI; NORBERTO, 2009; MOURA, 2006).

Os efeitos benéficos da AHT são atribuídos aos antígenos presentes no sangue, os quais estimulam a produção de anticorpos quando o sangue é injetado no músculo (VADJA et al., 1967). Além de apresentar efeitos em doenças auto-imunes, a AHT pode ser utilizada como auxiliar ao tratamento convencional em doenças infecciosas, devido à observação da presença de derivados das bactérias do foco de infecção na corrente sanguínea durante a fase ativa da doença (ROSENOW, 1914). A AHT atua complementando a antibioticoterapia, inibindo a reprodução dos microrganismos,

DOI: https://doi.org/10.25110/arqsaude.v19i2.2015.5430

${ }^{1}$ Farmacêutico. Laboratório Reunidos de Umuarama-PR, Umuarama-PR, Brasil

${ }^{2}$ Farmacêutica. Doutora em Ciências Farmacêuticas. Docente da Universidade Federal de Mato Grosso do Sul (UFMS), Coxim-MS, Brasil.

${ }^{3}$ Médico. Especialista em Pediatria. Umuarama, PR, Brasil.

${ }^{4}$ Farmacêutica. Doutora em Imunologia Básica e Aplicada. Docente da Universidade Estadual de Maringá (UEM), Maringá-PR, Brasil.

Endereço para correspondência: Universidade Estadual de Maringá, Centro de Ciências da Saúde, Departamento de Análises Clínicas e Biomedicina. Av. Colombo, 5790 - LEPAC - Laboratório de Imunologia Clínica. Cidade Universitária. CEP: 87020900, Maringá-PR, Brasil, telefone: (44) 30114878, Ramal: 4878; e-mail: smaaarraes@uem.br 
enquanto o sistema imunológico ativado derrota a infecção (TEIXEIRA, 1940).

Por ser um procedimento simples, seguro e barato (BOCCI, 1994), a AHT é amplamente procurada, principalmente em laboratórios e farmácias, despertando interesse de pacientes portadores de doenças crônico degenerativas, em especial as auto-imunes, que apresentam pouca ou nenhuma melhora em seus quadros clínicos com os tratamentos tradicionais. A AHT vem sendo extensamente usada em uma variedade de doenças e condições. Embora, no passado, tenha sido usada quase que empiricamente (GEOVANINI; NORBERTO, 2009), alguns estudos buscam elucidar suas ações, principalmente nas doenças auto-imunes (TEIXEIRA, 1940; ALVORD; SHAW; HRUBY, 1979; WILLIANS, 1990; BOCCI, 1994; RIVA et al., 1995; WEBSTER et al., 2000; HERNÁNDEZ et al., 2001; TYLICKI et al., 2001; GEOVANINI; JÚNIOR; PALERMO, 2007; GEOVANINI; NORBERTO, 2009).

A estimulação do sistema imunológico pela AHT foi avaliada em experimentos com humanos e animais (TEIXEIRA, 1940; SANTIN; BRITO, 2006). No entanto não foram encontrados trabalhos com AHT em humanos hígidos, e a maioria dos estudos achados na literatura consultada são antigos. Atualmente é perceptível a grande repercussão da AHT via internet, entre cidadãos de várias partes do mundo como Holanda, Portugal, Espanha, países da América Latina, Estados Unidos e Canadá. A técnica só poderá ser utilizada com segurança e tornar-se viável ou não, quando for esclarecida após estudos, a existência de eficácia e inocuidade. Há por isso a necessidade de se realizar pesquisas para compreensão dos mecanismos, como o proposto neste trabalho. Somente deste modo poder-se-á demonstrar sua utilidade e sua eficácia ou não em melhorar a imunidade. Na ausência destes estudos, as pessoas certamente continuarão praticando a AHT, mesmo sem qualquer esclarecimento técnico, baseado em evidências clínicas.

O objetivo deste estudo foi avaliar o efeito do tratamento pela auto-hemoterapia na estimulação da resposta imune, por meio de análise dos níveis de imunoglobulinas no soro e de monócitos no sangue de indivíduos saudáveis que realizaram pela primeira vez o tratamento.

\section{Material e Método}

\section{Aspectos Éticos}

Todo o procedimento foi previamente aprovado pelo Comitê Permanente de Ética em Pesquisa Envolvendo Seres Humanos da Universidade Estadual de Maringá (COPEP/UEM; Parecer número 101/2009).

\section{Participantes}

Foi selecionado um grupo de dez pessoas saudáveis (P1-P10), que passaram por uma avaliação clínica realizada por um médico a fim de examinar a saúde geral de cada participante. O grupo foi composto de cinco homens e cinco mulheres, com idade entre 20 e 50 anos, que se dispusera a participar da pesquisa e que ainda não haviam realizado AHT. A seleção do grupo foi aleatória, pelo sorteio dos participantes a partir de uma lista de candidatos voluntários de um Laboratório de Análises Clínicas do interior do Paraná, Brasil.

\section{Auto-hemoterapia}

A AHT foi realizada por um médico treinado em um Laboratório de Análises Clínicas, a técnica foi realizada a partir da retirada de $10 \mathrm{~mL}$ de sangue venoso do voluntário e em seguida aplicada no músculo do mesmo, com a utilização de seringas e agulhas estéreis. A região escolhida para aplicação do sangue foi o quadrante látero-superior do músculo glúteo máximo, o qual possui poucos nervos sensoriais, permitindo uma administração menos dolorosa e mais segura. Foi realizada apenas uma aplicação em cada participante.

\section{Material biológico}

Um dia antes da AHT e nove dias consecutivos após a aplicação (sempre no mesmo horário) da AHT foi retirado cerca de $10 \mathrm{~mL}$ de sangue de cada participante, sendo que três mililitros foram coletados com anticoagulante (ácido etilenodiaminotetracético) e utilizado para realização do hemograma para posterior contagem dos monócitos. O restante foi deixado coagular e separado o soro para realização da pesquisa dos níveis de imunoglobulinas.

\section{Contagem de monócitos}

A contagem do número de monócitos foi realizada pelo diferencial de leucócitos em esfregaço sanguíneo corado com uma mistura de May-Grunwald-Giemsa.

\section{Dosagem de Imunoglobulinas}

As dosagens de imunoglobulinas séricas imunoglobulina A ( $\operatorname{IgA}$ ), imunoglobulina $\mathrm{G}$ ( $\mathrm{IgG}$ ) e imunoglobulina $\mathrm{M}$ (IgM) foram realizadas pelo método Nefelometria, utilizando o kit da Beckman pelo aparelho Image Beckman Coulter. A imunoglobulina E (IgE) foi dosada pelo método Immunocap, Fluoroenzima imunoensaio, utilizando o Kit Phadia no aparelho Licap 1000. Os valores de referência utilizados foram: IgA: 82 a $453 \mathrm{mg} / \mathrm{dL}$; IgG: 751 a $1560 \mathrm{mg} / \mathrm{dL}$; IgM: 46 a $304 \mathrm{mg} / \mathrm{dL}$; IgE: até $140 \mathrm{KU} / \mathrm{L}$.

\section{Análise estatística}

Os dados foram inicialmente submetidos ao teste Shapiro-Wilk para verificação do tipo de distribuição. Dados com distribuição normal foram expressos como média \pm desvio-padrão, e os de distribuição livre foram expressos como mediana e percentis 25 e 75 (P25; P75). Para comparação de dados antes e após AHT, utilizou-se teste t de Student para amostras relacionadas (para dados que tinham distribuição normal) e Wilcoxon (para os que tinham distribuição livre). Foi considerado estatisticamente significante valores de $p$ menores que 0,05. Para realização das análises foi utilizado programa Bioestat 5.0.

\section{Resultados}

De forma geral, homens e mulheres responderam de 
forma semelhante às variações do número de monócitos e a concentração das imunoglobulinas, independentemente da idade. Porém, a variação no número de monócitos foi grande entre os participantes. Dos dez participantes analisados, nove apresentaram aumento do número de monócitos, que variou de $17 \%$ (menor aumento) a $250 \%$ (maior aumento), conforme apresentado na tabela 1 . No entanto um dos participantes (P9) apresentou redução de 75\% nos monócitos.

Tabela 1: Variação da porcentagem de monócitos para cada participante antes da auto-hemoterapia (AHT) e seu respectivo dia de maior aumento

\begin{tabular}{cccc}
\hline Participantes & $\begin{array}{c}\mathbf{N}^{\mathbf{0}} \text { de monócitos antes da } \\
\text { AHT }\end{array}$ & $\begin{array}{c}\text { \% de aumento de } \\
\text { monócitos }\end{array}$ & Dia do maior aumento \\
\hline P1 & 4 & 75 & $8^{\circ}$ dia após AHT \\
P2 & 2 & 250 & $7^{\circ}$ dia após AHT \\
P3 & 5 & 20 & $2^{\circ}$ dia após AHT \\
P4 & 5 & 20 & $3^{\circ}$ dia após AHT \\
P5 & 6 & 17 & $7^{\circ}$ dia após AHT \\
P6 & 5 & 20 & $1^{\circ}$ dia após AHT \\
P7 & 2 & 200 & $1^{\circ}$ dia após AHT \\
P8 & 2 & 100 & $4^{\circ}$ dia após AHT \\
P9 & 4 & $0^{*}$ & Até $^{\circ}$ dia após AHT \\
P10 & 3 & 100 & $9^{\circ}$ dia após AHT \\
\hline
\end{tabular}

* Não houve aumento do número de monócitos.

Foi observado aumento da IgM em nove dos indivíduos estudados, que variou de 10 a $28 \%$. O aumento da IgG foi demonstrado em todos os indivíduos e variou de 13 a 36\% (Tabela 2). Houve um aumento de 3 a $89 \%$ da IgA em nove indivíduos e também aumento de 3 a $88 \%$ da $\operatorname{IgE}$ em oito deles (Tabela 3). Foi observado que nos participantes que não apresentaram aumento de imunoglobulinas os níveis foram mantidos.

Tabela 2: Variação da porcentagem das imunoglobulinas M e G (IgM e IgG) para cada participante antes da auto-hemoterapia (AHT) e seu respectivo dia de maior aumento.

\begin{tabular}{|c|c|c|c|}
\hline Participantes & Imunoglobulina & $\begin{array}{l}\text { \% de aumento das } \\
\text { Imunoglobulinas }\end{array}$ & Dia do maior aumento \\
\hline \multirow[t]{2}{*}{$\mathrm{P} 1$} & $\operatorname{IgM}$ & 20 & $8^{\circ}$ dia após AHT \\
\hline & $\mathrm{IgG}$ & 36 & $8^{\circ}$ dia após AHT \\
\hline \multirow[t]{2}{*}{$\mathrm{P} 2$} & $\operatorname{IgM}$ & 28 & $7^{\circ}$ dia após AHT \\
\hline & $\operatorname{IgG}$ & 13 & $6^{\circ}$ dia após AHT \\
\hline \multirow[t]{2}{*}{ P3 } & $\operatorname{IgM}$ & 16 & $7^{\circ}$ dia após AHT \\
\hline & $\operatorname{IgG}$ & 19 & $6^{\circ}$ dia após AHT \\
\hline \multirow[t]{2}{*}{$\mathrm{P} 4$} & $\operatorname{IgM}$ & 11 & $7^{\circ}$ dia após AHT \\
\hline & $\operatorname{IgG}$ & 15 & $6^{\circ}$ dia após AHT \\
\hline \multirow[t]{2}{*}{ P5 } & $\operatorname{IgM}$ & 10 & $9^{\circ}$ dia após AHT \\
\hline & $\operatorname{IgG}$ & 20 & $9^{\circ}$ dia após AHT \\
\hline \multirow[t]{2}{*}{ P6 } & $\operatorname{IgM}$ & 11 & $2^{\circ}$ dia após AHT \\
\hline & $\operatorname{IgG}$ & 20 & $2^{\circ}$ dia após AHT \\
\hline \multirow[t]{2}{*}{ P7 } & $\operatorname{IgM}$ & 9 & $6^{\circ}$ dia após AHT \\
\hline & $\operatorname{IgG}$ & 8 & $6^{\circ}$ dia após AHT \\
\hline \multirow[t]{2}{*}{ P8 } & $\operatorname{IgM}$ & 8 & $5^{\circ}$ dia após AHT \\
\hline & $\operatorname{IgG}$ & 12 & $2^{\circ}$ dia após AHT \\
\hline \multirow[t]{2}{*}{ P9 } & $\operatorname{IgM}$ & $0 *$ & Até o $9^{\circ}$ dia após AHT \\
\hline & $\operatorname{IgG}$ & 5 & $9^{\circ}$ dia após AHT \\
\hline \multirow[t]{2}{*}{$\mathrm{P} 10$} & $\operatorname{IgM}$ & 10 & $3^{\circ}$ dia após AHT \\
\hline & $\mathrm{IgG}$ & 16 & $3^{\circ}$ dia após AHT \\
\hline
\end{tabular}


Tabela 3: Variação da porcentagem das imunoglobulinas A e E (IgA; IgE) para cada participante antes da auto-hemoterapia (AHT) e seu respectivo dia de maior aumento.

\begin{tabular}{|c|c|c|c|}
\hline Participantes & Imunoglobulina & $\begin{array}{l}\text { \% de aumento das } \\
\text { Imunoglobulinas }\end{array}$ & Dia do maior aumento \\
\hline \multirow[t]{2}{*}{$\mathrm{P} 1$} & $\operatorname{Ig} \mathrm{A}$ & 10 & $6^{\circ}$ dia após AHT \\
\hline & $\operatorname{IgE}$ & 23 & $3^{\circ}$ dia após AHT \\
\hline \multirow[t]{2}{*}{$\mathrm{P} 2$} & $\operatorname{Ig} \mathrm{A}$ & 50 & $7^{\circ}$ dia após AHT \\
\hline & $\operatorname{IgE}$ & 17 & $7^{\circ}$ dia após AHT \\
\hline \multirow[t]{2}{*}{$\mathrm{P} 3$} & $\operatorname{Ig} \mathrm{A}$ & 4 & $6^{\circ}$ dia após AHT \\
\hline & $\operatorname{IgE}$ & 3 & $8^{\circ}$ dia após AHT \\
\hline \multirow[t]{2}{*}{$\mathrm{P} 4$} & $\operatorname{Ig} \mathrm{A}$ & 7 & $6^{\circ}$ dia após AHT \\
\hline & $\operatorname{IgE}$ & 29 & $6^{\circ}$ dia após AHT \\
\hline \multirow[t]{2}{*}{ P5 } & $\operatorname{Ig} \mathrm{A}$ & 89 & $9^{\circ}$ dia após AHT \\
\hline & $\operatorname{IgE}$ & 8 & $9^{\circ}$ dia após AHT \\
\hline \multirow[t]{2}{*}{ P6 } & $\operatorname{Ig} \mathrm{A}$ & 3 & $4^{\mathrm{o}}$ dia após AHT \\
\hline & $\operatorname{IgE}$ & 88 & $5^{\circ}$ dia após AHT \\
\hline \multirow[t]{2}{*}{$\mathrm{P} 7$} & $\operatorname{Ig} \mathrm{A}$ & 3 & $8^{\circ}$ dia após AHT \\
\hline & $\operatorname{IgE}$ & 29 & $7^{\circ}$ dia após AHT \\
\hline \multirow[t]{2}{*}{ P8 } & $\operatorname{Ig} \mathrm{A}$ & 13 & $2^{\circ}$ dia após AHT \\
\hline & $\operatorname{IgE}$ & 15 & $1^{\circ}$ dia após AHT \\
\hline \multirow[t]{2}{*}{ P9 } & $\operatorname{Ig} \mathrm{A}$ & $0 *$ & Até o $9^{\circ}$ dia após AHT \\
\hline & $\operatorname{IgE}$ & $0 *$ & Até o $9^{\circ}$ dia após AHT \\
\hline \multirow[t]{2}{*}{$\mathrm{P} 10$} & $\operatorname{IgA}$ & 8 & $3^{\circ}$ dia após AHT \\
\hline & $\operatorname{IgE}$ & $0 *$ & Até o $9^{\circ}$ dia após AHT \\
\hline
\end{tabular}

* Não houve aumento.

Foi demonstrado aumento significante na comparação do número de monócitos $(\mathrm{p}=0,0016)$ e das imunoglobulinas $M(p=0,0024), G(p=0,0001)$ e $E(p=0,00117)$ antes da AHT e após aplicação da AHT, a IgA não mostrou diferença significativa, conforme apresentado na tabela 4.

Tabela 4: Média \pm desvio padrão do número de monócitos e das dosagens de imunoglobulinas M, G, A, mediana e percentis 25 e 75 (P25; P75) da imunoglobulina E antes da auto-hemoterapia (AHT) e o maior aumento após AHT.

\begin{tabular}{lccccc}
\hline & Monócitos & IgM & IgG & IgA & IgE \\
\hline Antes AHT & $3,78^{\mathrm{a}} \pm 1,56$ & $107,78^{\mathrm{b}} \pm 55,24$ & $1136,8^{\mathrm{c}} \pm 298,18$ & $226,66 \pm 96,00$ & $\begin{array}{c}151,00^{\mathrm{d}} \\
(51,75 ; 38,40)\end{array}$ \\
$\begin{array}{l}\text { Após AHT } \\
\text { (maior aumento) }\end{array}$ & $6,12^{\mathrm{a}} \pm 0,92$ & $125,55^{\mathrm{b}} \pm 61,95$ & $1309,0^{\mathrm{c}} \pm 300,47$ & $259,77 \pm 137,16$ & $157,00^{\mathrm{d}}$ \\
\hline Valores segidos & & & & $(61,00 ; 44,57)$ \\
\hline
\end{tabular}

\section{Discussão}

Após aplicação da AHT houve aumento médio de monócitos de $62 \%(p=0,0016)$. Os resultados encontrados corroboram com os achados de Teixeira (1940) que demonstrou estimulação e liberação de células de defesa do organismo e um aumento expressivo de monócitos em mais de cinco vezes (de $5 \%$ para mais de $22 \%$ ). Como a AHT busca potencializar o sistema imunológico, estimulando e liberando células de defesa, entre elas o monócito (CHOPRA; CHOPRA, 1995), os resultados sugerem que a AHT foi capaz de influenciar de alguma forma o sistema imunológico dos participantes. De acordo com Veronesi (1976), os macrófagos se originam de monócitos da medula óssea, de onde são lançados na corrente sanguínea, para colonizar os tecidos e órgãos.

$\mathrm{O}$ aumento de monócitos também foi evidenciado anteriormente, numa média geral de $37,5 \%$ após o segundo dia e $54,1 \%$ após o quinto dia da realização da AHT (ROSIN, 2007). Em nosso estudo, o aumento de monócitos variou de indivíduo para indivíduo, onde foi observado aumento do segundo até o último dia de análise ( $\left.9^{\circ} \mathrm{dia}\right)$. Esta variação demonstra que cada participante respondeu de forma diferente ao estímulo aplicado. O maior aumento de monócitos encontrado neste trabalho foi de $250 \%$ e no trabalho anteriormente citado foi de 142,8\%. Essa diferença possivelmente se deve ao fato de que o autor avaliou a porcentagem de monócitos apenas até o quinto dia após aplicação e o aumento de $250 \%$ observado neste estudo, ocorreu após o sétimo dia de aplicação. Descrições na literatura buscam elucidar o mecanismo da AHT, para Teixeira (1940) o aumento se deve ao fato de que o sangue extraído por punção venosa é um sangue asfíxico que, por curto lapso, se põe em contato com um corpo estranho (seringa), o que é suficiente para provocar modificações físico-químicas e, por isso, quando injetado no orga- 
nismo, atua como uma proteína estranha, agindo dessa forma a produzir estímulos quando o sangue é injetado no músculo.

Os macrófagos desempenham papel chave na regulação do sistema imunológico, participando das repostas imune inata e específica. $\mathrm{O}$ aumento dos monócitos observados neste estudo sugere que houve um estímulo da resposta imune, pois essas células fazem a homeostase local e sistêmica por meio de uma complexa série de eventos celulares e bioquímicos (SABET et al., 1977). O seu aumento pelo estímulo da auto-hemoterapia, é de grande valia uma vez que doenças infecciosas, alérgicas, corpos estranhos e obstruções de vasos sanguíneos podem ser combatidos por estas células, pois são capazes de vencer os estados patológicos ou pelo menos minimizá-los (RAVAUT, 1913).

Em um dos pacientes avaliados não foi observado aumento do número de monócitos. Para melhor compreendermos o que ocorreu com este paciente seria necessário fazer uma nova avaliação.

O aumento da IgM foi observado em todos os participantes, possivelmente por representar uma das primeiras respostas imunológicas. Esse aumento foi de 16\% ( $\mathrm{p}=$ $0,0024)$ quando calculada a média para todos os indivíduos. A IgM é a primeira imunoglobulina circulante produzida pelo plasmócito durante a resposta imunológica. Sua elevação ocorre logo após o primeiro contato com o antígeno, é bastante eficiente na fixação de complemento, por possuir a capacidade de iniciar a cascata deste sistema juntamente com a IgG (CHINEN; SHEARER, 2005). Também foi observada elevação da $\operatorname{IgG}$ de $15 \%(p=0,0001)$, esta imunoglobulina aparece numa fase mais tardia da exposição ao agente agressor, mostrando que tanto a resposta inicial como a mais tardia foi ativada.

Observamos uma elevação dos níveis séricos da IgA de 3 a $89 \%$, porém quando foi calculada a média de todos os indivíduos não houve diferença significativa após a aplicação da AHT. A IgA inibe a aderência dos microrganismos à superfície das células da mucosa e impede a penetração nos tecidos orgânicos, e quando agregada liga-se aos leucócitos polimorfonucleares podendo ativar a via alternativa do complemento, fornecendo um mecanismo extra de proteção. A IgA tem papel importante na neutralização e eliminação de antígenos locais e na modulação de fatores imunológicos teciduais ou humorais (STOKES; SOOTHILL; TURNER, 1975). Como o estudo foi realizado com indivíduos hígidos, possivelmente o complexo sistema de proteção local contra agentes infecciosos poderia estar envolvido em outras situações de defesa que exige a presença de componentes não reconhecidos como pertencentes ao indivíduo (SOUZA; LEHN; DENARDIN, 2003).

Os níveis normais de IgE circulantes são extremamente baixos em comparação com outras imunoglobulinas. A IgE é única em determinados aspectos estruturais e na função que desempenha nas doenças alérgicas. Os níveis elevados são normalmente observados nos casos de doenças alérgicas, infecções parasitárias, aspergilose pulmonar, síndrome de Wiskott-Aldrich e mieloma (KAIREMO; LINDBERG; PRYTZ, 1999; VILLARREAL; VILLARREAL; DOMINGO, 1999). O aumento da IgE foi de 3 a $88 \%$ em oito indivíduos e a mediana para todos os pacientes representou um aumento de 4\% ( $\mathrm{p}=0,00117)$. No entanto, quatro amostras apresentaram níveis elevados dessa imunoglobulina antes mesmo do tratamento com AHT e dois pacientes avaliados apresentavam níveis abaixo do referencial. A variação observada pode ser devido ao resultado da dieta alimentar, antecedentes genéticos, localização geográfica e outros fatores. Por essa razão, a IgE não deve ser avaliado de forma isolada, deve ser utilizada em conjunto com outras imunoglobulinas.

Quando o organismo humano ou animal é agredido por agentes infecciosos ou parasitários, é acionado o sistema imunitário em seus vários compartimentos, a fim de destruir ou neutralizar o agressor. Tanto a imunidade mediada por células, como a mediada por plasmócitos, produtores das imunoglobulinas (VERONESI, 1976), são complementadas pelos macrófagos, para impedir a ação patogênica do agente invasor. Baseado nesses achados acredita-se que se o estudo fosse realizado em indivíduos com alguma doença, os resultados poderiam ser mais expressivos, pois haveria uma maior ativação do sistema imunológico, uma vez que a AHT tem mostrado resultados satisfatórios em vários procedimentos como tratamento de feridas (GEOVANINI; JÚNIOR; PALERMO, 2007; GEOVANINI; NORBERTO, 2009), profilaxia em pacientes pré-cirúrgicos de forma a prevenir complicações pulmonares pós-operatórias (TEIXEIRA, 1940), artrite reumatóide (HERNÁNDEZ et al., 2001) e tratamento de pacientes poliartríticos crônicos, com aumento da fagocitose de Staphylococus aureus e Streptococus pyogenes uma hora após a realização da técnica, permanecendo até o término de todo o tratamento (VADJA et al., 1967). Além de resultados promissores na medicina veterinária (SANTIN; BRITO, 2006). Segundo Rocha et al. (2007), quando o sistema imunitário é adequadamente ativado, o hospedeiro atinge um "estado de imunidade" que o torna mais apto à sobrevivência. Portanto, são necessários mais estudos para melhor avaliar os efeitos da auto-hemoterapia, como na avaliação da resposta imune celular por meio de pesquisa de células como linfócitos e citocinas.

Vale destacar que o Plasma Rico em Plaquetas (PRP) tem sido bastante utilizado (VENDRAMIN et al., 2006). Estudos mostram um grande potencial de melhorar os resultados em diversos procedimentos ortopédicos, neurocirúrgicos e de cirurgia plástica (BHANOT; ALEX, 2002; MAZZUCCO et al., 2004). O que difere a AHT da PRP é a forma que o sangue é tratado após coleta para reaplicação no paciente, no entanto o principio é semelhante, em ambas as situações é utilizado sangue do próprio paciente com resultados benéficos após reaplicação do mesmo.

Cabe ainda ressaltar que a AHT é questionada no que se refere a sua comprovação científica (LEITE; BARBOSA; GARRAFA, 2008). É natural que haja este processo de resistência. Outras terapias alternativas que no passado foram motivos de polêmica, hoje são reconhecidas e até recomendadas. Como exemplo, temos a homeopatia e acupuntura. A aceitação da eficácia dessas terapias alternativas vem ocorrendo em larga medida, independentemente do progresso do conhecimento médico sobre os seus mecanismos de ação. A constatação de sua efetividade e eficácia, por parte de pacientes e terapeutas, tem sido o principal fator a motivar sua adoção e expansão nos serviços e nas instituições de atenção à saúde (NASCIMENTO, 1998). Dessa forma, faz-se necessário uma melhor elucidação da técnica da AHT, constituindo objeto de investigação científica para diversos pesquisadores. 


\section{Conclusão}

O aumento do número de monócitos e das imunoglobulinas mostra que o tratamento com auto-hemoterapia foi capaz de estimular a resposta imunológica dos participantes. No entanto, a sua aplicabilidade deve aguardar outros estudos complementares.

\section{Referências}

ALVORD, E. C.; SHAW, C. M.; HRUBY, S.

Autohaemotherapy Approaches to the treatment of central nervous system autoimmune disease. Ann. Neurology, v. 6, p. 469-473, 1979.

BHANOT, S.; ALEX, J. C. Current applications of platelet gels in facial plastic surgery. Facial Plast. Surg. v. 18, n. 1, p. 27-33, 2002.

BOCCI, V. Autohaemotherapy after treatment of blood with ozone. A reappraisal. J. Int. Med. Res. v. 22, p.131-144, 1994.

CHOPRA, A.; CHOPRA, M. D. Autohaemotherapy in chronic urticaria. Indian. J. Dermatol. Venereol. Leprol. v. 61. n. 5. p. 323-324, 1995.

CHINEN, J.; SHEARER, W. T. Basic and clinical immunology. J. Allergy. Clin. Immunol. v. 116, n. 2. p. 411-418, 2005.

GEOVANINI, T.; NORBERTO, M. M. C. Tratamento da esclerodermia doença auto imune através da autohemoterapia: um estudo de caso clínico. Rev. Enf. Ref. v. 2, n. 9, p. 51-59, 2009.

GEOVANINI, T.; OLIVEIRA JÚNIOR, A. G. O.; PALERMO, T. C. S. Manual de curativos. São Paulo: Corpus, 2007. p. 81-98.

HERNÁNDEZ, M. L. et al. Autohemoterapia:¿ alternativa eficaz en la patología autoinmune?. Atención Primaria, v. 28, n. 4, p. 291-292, 2001.

KAIREMO, K. J.; LINDBERG, M.; PRYTZ, M. IgE myeloma: a case presentation and a review of the literature. Scand. J. Clin. Lab. Invest. v. 59, n. 6, p. 451-456, 1999.

LEITE, D. F.; BARBOSA, P. F. T.; GARRAFA, V. Autohemoterapia, intervenção do estado e bioética. Rev. Assoc. Med. Bras. v. 54, n. 2, p. 183-88, 2008.

MAZZUCCO, L. et al. The use of autologous platelet gel to treat difficult-to-heal wounds: a pilot study. Transfusion. v. 44, n.7, p. 1013-1018, 2004.

METTENLEITER, M. W. Autohemotransfusion in preventing postoperative lung complications. Am. J. Surg. v. 22, n. 2, p. 321-323, 1936.

MOURA, L. Auto-hemoterapia. 2006. Disponível em:
$<$ http://inforum.insite.com.br>. Acesso em: 15 fev. 2014.

NASCIMENTO, M. C. Da panacéia mística a especialidade médica: a acupuntura na visão da imprensa escrita. Hist. Cienc. Saúde-Manguinhos, v. 5, n. 1, p. 99-113, 1998.

RAVAUT, M. Paul Essai sur L'Autoh, matothrapie dans Quelques Dermatoses. Ann. Derm. Syph. v. 4, p. 292-296, 1913.

REIMANN, H. A.; HAVENS W. P. Focal infection and systemic disease: a critical appraisal. JAMA. v. 114, n.1, p. $1-6,1990$.

RIVA, S. E. et al. Effects of ozonized autohaemotherapy on human hair cycle. Panminerva med. v. 37, n.3, p. 129-132, 1995.

ROCHA, K. C.; GORESCU, R.; BELTRAME, R. L. Metodologia laboratorial para estudo da resposta imune celular. In: VAZ, A. J.; TAKEI, K.; BUENO, E. C. Ciências farmacêuticas imunoensaios - fundamentos e aplicações. Rio de Janeiro: Guanabara Koogan, 2007. p. 119-131.

ROSENOW, E. C. Mouth infection as a source of systemic disease. JAMA, v. 63, n. 23, p. 2024-2025, 1914.

ROSIN, M. Variação da porcentagem de monócitos no sangue circulante antes e após a auto-hemoterapia. 2007. Monografia (Especialização em Hematologia Laboratorial) - Academia de Ciência e Tecnologia, São José do Rio Preto, 2007.

SABET, T. et al. A simple method for obtaining peritoneal macrophages from chickens. J. Immunol. Methods, v. 14, n. 2, p. 103-110, 1977.

SANTIN, A. P. I.; BRITO, L. A. B. Estudo da papilomatose cutânea em bovinos leiteiros: comparação de diferentes tratamentos. Ciênc. Anim. Bras. v. 5, n. 1, p. 41-47, 2006.

SOUZA, M. R.; LEHN, C. N.; DENARDIN, O. V. P. Níveis sérico e salivar de imunoglobulina a em portadores de câncer da boca e orofaringe. Rev. Assoc. Med. Bras. v. 49, n. 1, p. 40-44, 2003.

STOKES, C. R.; SOOTHILL, J. F.; TURNER, M. W. Immune exclusion is a function of IgA. Nature, v. 26, n. 255, p. 745-746, 1975.

TEIXEIRA, J. Complicações pulmonares pós-operatóriasautohemotransfusão. Rev. Bras. Cir. v. 2, n. 3, p. 213-230, 1940 .

TYLICKI, L. et al. Beneficial clinical effects of ozonated autohemotherapy in chronically dialysed patients with atherosclerotic ischemia of the lower limbs-pilot study. Int. J. Artif. Organs. v. 24. n. 2, p. 79-82, 2001.

VADJA, G. et al. Data concerning the mechanism of autohaemotherapy in pllyarthritic patients. $\mathbf{Z}$. 
Immunitatsforsch Allerg Klin Immunol. v. 133, n. 1, p. $1-6,1967$.

VENDRAMIN, F. S. et al. Plasma rico em plaquetas e fatores de crescimento: técnica de preparo e utilização em cirurgia plástica. Rev. Col. Bras. Cir. v. 33, n. 1, p. 24-28, 2006 .

VERONESI, R. Imunoterapia: o impacto médico do século. Med. Hoje, v. 2, n. 13, p. 148-152, 1976.

VILLARREAL, O.; VILLARREAL, J. J.; DOMINGO, J. A. Progressive eosinophilia and elevated IgE in enterobiasis, Allergy. v. 54, n. 6, p. 646-648, 1999.

WEBSTER, G. J. et al. Molecular epidemiology of a large outbreak of hepatitis B linked to autohaemotherapy. Lancet. v. 356, n. 9227, p. 379-384, 2000.

WILLIANS, J. et al. Autohaemotherapy for genital, anal and perianal warts. Indian J. Sex. Transm. Dis. v. 11, n. 2, p. $57-58,1990$. 